Isabella, Silvia S.L., Urbain, Charline, Cheyne, James Allan, \& Cheyne, Douglas

Pupillary responses and reaction times index different cognitive processes in a combined Go/Switch incidental learning task

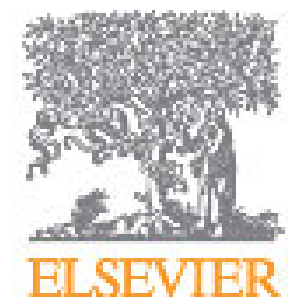 \\ Article. Version publiée - Published version. (c) ELSEVIER
}

\section{Citation APA:}

DOI: 10.1016/j.neuropsychologia.2019.02.007

\section{Also available at: http://hdl.handle.net/2013/ULB-DIPOT:oai:dipot.ulb.ac.be:2013/284945}

Cet article publié par ELSEVIER provient du Dépôt institutionnel de l’Université Libre de Bruxelles, DIfusion http://difusion.ulb.ac.be. Il n'est accessible qu'aux membres de la communauté universitaire de l'ULB sur le réseau sécurisé de l'ULB.

Tout utilisateur autorisé peut lire, télécharger ou reproduire cet article à des fins d'usage privé ou à des fins non commerciales d'enseignement ou de recherche scientifique. Il ne peut être atteint à l'intégrité de l'article, et les noms des auteurs et de l'éditeur doivent être conservés. Tout téléchargement systématique des articles publiés par ELSEVIER mis à disposition dans DI-fusion est interdit.

This article published by ELSEVIER comes from the Institutional repository of Universite Libre de Bruxelles, DI-fusion http://difusion.ulb.ac.be. It is accessible only to the members of the university community of ULB on the ULB secure network.

Any authorized user may read, download or reproduce this article for private usage, or for non commercial research or educational purposes. The integrity of the article and identification of the author and copyright owner must be preserved. Systematic downloading of articles published by ELSEVIER that are available in DI-fusion is not permitted. 


\title{
Pupillary responses and reaction times index different cognitive processes in a combined Go/Switch incidental learning task
}

\author{
Silvia L. Isabella ${ }^{\mathrm{a}, \mathrm{b}}$, Charline Urbain ${ }^{\mathrm{c}, \mathrm{d}}$, J. Allan Cheyne ${ }^{\mathrm{e}}$, Douglas Cheyne ${ }^{\mathrm{a}, \mathrm{b}, \mathrm{f}, *}$ \\ ${ }^{\text {a }}$ Program in Neurosciences and Mental Health, Hospital for Sick Children Research Institute, Toronto, Canada \\ ${ }^{\mathrm{b}}$ Institute of Medical Sciences and Institute of Biomaterials and Biomedical Engineering, University of Toronto, Toronto, Canada \\ ${ }^{\mathrm{c}}$ Neuropsychology and Functional Neuroimaging Research Group at Center for Research in Cognition and Neurosciences (CRCN) and ULB Neurosciences Institute (UNI), \\ Brussels, Belgium \\ ${ }^{\mathrm{d}}$ Laboratoire de Cartographie fonctionnelle du Cerveau, UNI, ULB, Brussels, Belgium \\ ${ }^{\mathrm{e}}$ Department of Psychology, University of Waterloo, Waterloo, Canada \\ ${ }^{\mathrm{f}}$ Department of Medical Imaging, University of Toronto, Toronto, Canada
}

\section{A R T I C L E I N F O}

\section{Keywords:}

Inhibitory control

Pupillometry

Cognitive load

Implicit learning

\begin{abstract}
A B S T R A C T
In previous studies we have provided evidence that performance in speeded response tasks with infrequent target stimuli reflects both automatic and controlled cognitive processes, based on differences in reaction time (RT) and task-related brain responses (Cheyne et al. 2012, Isabella et al. 2015). Here we test the hypothesis that such shifts in cognitive control may be influenced by changes in cognitive load related to stimulus predictability, and that these changes can be indexed by task-evoked pupillary responses (TEPR). We manipulated stimulus predictability using fixed stimulus sequences that were unknown to the participants in a Go/Switch task (requiring a switch response on $25 \%$ of trials) while monitoring TEPR as a measure of cognitive load in 12 healthy adults. Results showed significant improvement in performance (reduced RT, increased efficiency) for repeated sequences compared to occasional deviant sequences (10\% probability) indicating that incidental learning of the predictable sequences facilitated performance. All behavioral measures varied between Switch and Go trials (RT, efficiency), however mean TEPR amplitude (mTEPR) and latency to maximum pupil dilation were particularly sensitive to Go/Switch. Results were consistent with the hypothesis that mTEPR indexes cognitive load, whereas TEPR latency indexes time to response selection, independent from response execution. The present study provides evidence that incidental pattern learning during response inhibition tasks may modulate several cognitive processes including cognitive load, effort, response selection and execution, which can in turn have differential effects on measures of performance. In particular, we demonstrate that reaction time may not be indicative of underlying cognitive load.
\end{abstract}

\section{Introduction}

It has long been known that increases in cognitive requirements are accompanied by changes in pupil dilation. A seminal study in 1966 reported that task-evoked pupillary responses (TEPR), such as pupil dilation, was proportional to increasing task demands during a working memory task (Kahneman and Beatty, 1966). The authors additionally found reduced TEPR reflected diminished processing load with practice. Although many differences in cognitive requirements may be observable through behavior, including RT and error rates, TEPR has been shown to be additionally sensitive to cognitive requirements in the absence of behavioral differences. TEPR is sensitive to word imagery, in the absence of any behavioral responses (Simpson and Paivio, 1966), and was also sensitive to differences in Go/No-Go probability ratios, even when RT remained constant (Richer et al., 1983). This was the first evidence for a difference between processing demands indexed by pupillary measures and RT. A follow-up study demonstrated that RT and TEPR were not correlated across single-trials: RT increased according to the Hick-Hyman law (Hick, 1952), whereas TEPR increased almost linearly related to response uncertainty (Richer and Beatty, 1987). RT and TEPR appeared to reflect different aspects of processing demands, in that RT may index processing latency, whereas TEPR may index processing load (Richer and Beatty, 1987). This TEPR-RT dissociation has been replicated for the Go/No-Go paradigm (Molen et al.,

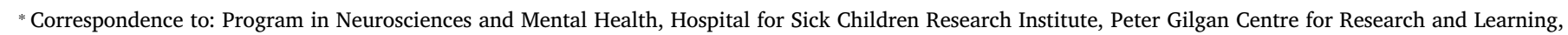
686 Bay St., Toronto, Ontario, Canada M5G 0A4.

E-mail address: douglas.cheyne@utoronto.ca (D. Cheyne). 
A

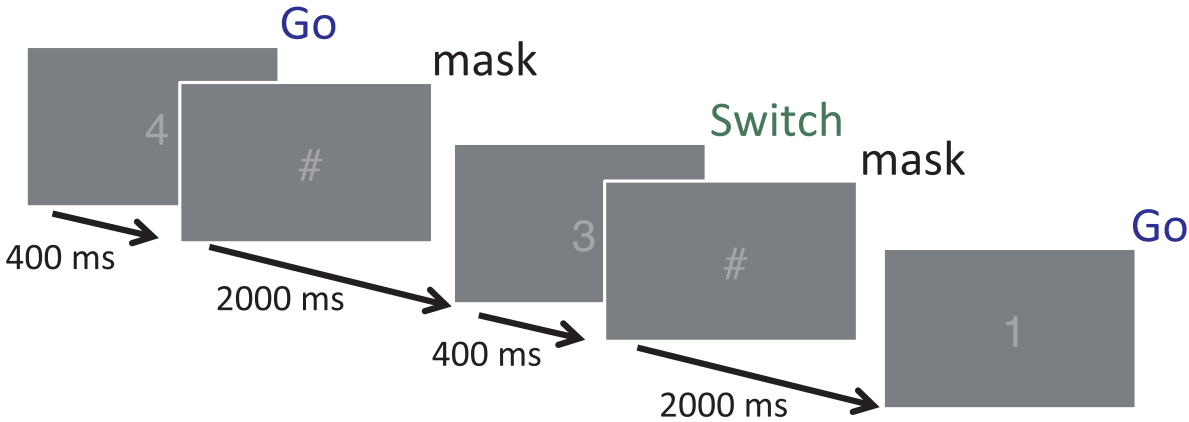

Fig. 1. Task design and required responses. (A) Digits were presented every $2400 \mathrm{~ms}$ for a duration of $400 \mathrm{~ms}$, followed by a stimulus mask ("\#") for a duration of $2000 \mathrm{~ms}$. Overall probability for each of the four stimuli was $25 \%$. (B) The correct response to the Switch stimulus (" 3 ") was a left index finger button press, whereas the correct response to all other stimuli was a right index finger button press.

B

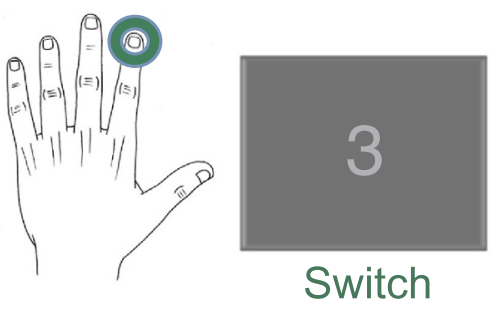

1989), as well as across disciplines during a translation task (Hyönä et al., 1995).

In a previous study, we found evidence for dissociation between reaction time (RT) and oscillatory brain signals related to cognitive control in two different response inhibition tasks involving rapidly presented frequent Go and rare No-Go or Switch cues (Isabella et al., 2015). Given that behavioral performance (RT and error rate) was highly similar across these two tasks, the observed differences in frontal theta $(4-8 \mathrm{~Hz})$ power during identical error responses were assumed to reflect covert differences between tasks. One possible factor may have been changing levels of cognitive control related to differences in response preparation, which is known to covary with stimulus probability (Gehring et al., 1992) and fluctuates over time (Seli, 2016). In such rapid response tasks, it is difficult to manipulate cognitive load directly without introducing additional working memory requirements, however ongoing fluctuations in cognitive load or attention during such tasks can be inferred by introspective report or can be quantified using pupillometry (Smallwood et al., 2011). Therefore, pupillometry can be an important tool for investigating covert differences between or within tasks. The objective of the current study was to investigate how various stimulus probabilities in a cognitive task effect cognitive load and behavioral responses, using TEPR as an indicator of cognitive load.

In the current study, we measured pupillary responses during performance of the response switching task (Cheyne et al., 2009) based on the Sustained Attention to Response Task (SART) (Robertson et al., 1997), a type of Go/No-Go paradigm that presents a frequent Go cue and a relatively rare Switch cue. In addition, we introduce a novel approach to varying cognitive load over the duration of the experiment by using fixed sequences of Go and Switch stimuli to induce incidental learning of the cues. Previous studies have shown that if the stimuli are presented in a patterned order, subjects may learn the pattern without conscious awareness (Jacoby and Witherspoon, 1982; Nissen and Bullemer, 1987). Furthermore, additional cognitive processing for trials may be required if deviations from the stimulus pattern are introduced, as response uncertainty would thereby be increased. This approach has the advantage of manipulating cognitive load over the course of the measurement through changes in target predictability without explicit manipulation of task difficulty that may be confounded with changes in working memory or other stimulus processing requirements.

Based on previous studies, we predicted that subjects would learn to anticipate Pattern Switch trials. This was directly contrasted with
Deviant Switch trials when the subject was presented with a Switch trial that was out of the pattern order, as well as Deviant Go trials that occurred when, according to the stimulus pattern, a Switch trial would normally occur. Such task variations parametrically vary the probability of an upcoming required response. Differences in RT and TEPR between pattern and deviant trials would provide evidence for learning of the patterns, while differences between RT and TEPR would provide evidence for a dissociation between reaction times and cognitive load in this task. Furthermore, we anticipated the possibility that once the pattern has been learned, there would be a difference in pre-stimulus pupil diameter, in anticipation of relatively easy Go trials over Switch trials, reflecting decreased attention to the task and a shift to a more automatic mode of responding as demonstrated in recent neuroimaging studies using the SART (Cheyne et al., 2012; Isabella et al., 2015). Similar to the SART, this task would also lend the possibility of combination with neuroimaging in future studies.

\section{Methods}

\subsection{Subjects}

Twelve healthy right-handed adults (7 females, range 22-29 years) participated in this experiment. All subjects were recruited from the Toronto area and provided informed consent using protocols approved by the Hospital for Sick Children Research Ethics Board. Subjects were compensated $40 \mathrm{CAD}$ for their participation.

\subsection{Task}

All subjects were presented with a rapid stream of digits from " 1 " to " 4 ", where each target has an equal $25 \%$ probability of occurrence. The rationale for this was to avoid inducing an oddball effect on any of the stimuli, particularly in combination with the switch or Deviant stimuli that would confound any pupillary effects on those trials (Squires et al., 1975). Each stimulus was displayed for $0.4 \mathrm{~s}$, followed by a stimulus mask ("\#") that was displayed for an additional $2 \mathrm{~s}$ until the presentation of the next digit, for a total inter-trial interval of $2.4 \mathrm{~s}$ (Fig. 1). The subjects were informed that they were performing a go-switch task, for which the default movement to stimuli 1, 2, or 4 was a button press with the right index finger, with instructions to switch response hands to the left index finger when presented with the target " 3 " stimulus. 
Subjects performed this task over 244 trials across each of 7 blocks. Each block began with 4 trials containing stimuli (digits 1-4) chosen at random. Subjects were uninformed that the remaining 240 stimuli were presented in 30 repeats of one of two 8-trial probabilistic sequences (sequence A: 3-1-4-3-2-4-1-2, sequence B: 1-4-2-3-4-1-3-2), known to induce pattern learning in typically developing children (aged 7-12 years) during a serial reaction time task (Gabriel et al., 2011). The two sequences were randomized across subjects, where a single sequence was presented for 7 successive blocks, except for the penultimate block when the alternate sequence was presented. The sequences were presented in this order with the objective of assessing any behavioral or physiological differences between sequence presentations. Stimuli for $90 \%$ of trials followed the sequence order (Pattern), whereas for the remaining $10 \%$ of trials, the stimulus for the individual trial did not follow the sequence order (Deviant).

All subjects were instructed to "respond as quickly as possible without committing too many errors". They were informed that they would receive feedback at the end of each block, with a $\$ 2$ performance reward for each block with overall RT under $0.4 \mathrm{~s}$ and error rate under $30 \%$ for right (Go) and left (Switch) responses. The target RT and error rate were based on empirically determined minimum performance values during pilot testing. Once the target RT and error rate were achieved, subjects were instructed to maintain or improve their scores in order to continue receiving the $\$ 2$ monetary reward for each block (for a maximum reward of \$14). This was done in order to maintain motivation for the duration of the experiment. Before commencing the test blocks, subjects performed a practice block consisting of the same stimuli presented in random order, for 64 trials lasting $2.5 \mathrm{~min}$. Subjects were told that they may repeat the practice block if desired. If the subject did not meet the $0.4 \mathrm{~s}$ RT and $30 \%$ error rate target during the practice, the experimenter explicitly asked the subject if she or he would like to perform another practice block. For the practice block only, feedback in the form of RT was presented on the left side of the screen after each response to help subjects determine a speed-accuracy strategy. Ahead of the test blocks, it was suggested to use the timing of the stimulus mask at $0.4 \mathrm{~s}$ as a benchmark for their RT on each trial.

Subjects were told that the goal of the study was to test the effect of a particular strategy, i.e., to covertly say the number as it appears on the screen. In addition to ensuring that subjects were naïve of the incidental learning task, during pilot testing we found that this strategy also improved learning of the sequence (data not shown). Following task performance on all 7 blocks, subjects were asked for general feedback on the task, whether they noticed any differences between blocks, and to write out a sample stream of stimuli from the experiment. This was in order to assess whether the presence of the sequence of stimuli was explicitly learned.

Notably, pilot testing preceded experimental data collection performed by 20 subjects (data not shown) in order to optimize many of the parameters used in this study. Some of the tested parameters include pre-task practice, instructions (suggested maximum reaction time for accuracy to exceed $70 \%$ ), the inter-trial interval (2.4 s), and the rate of deviant stimuli (10\%).

\subsection{Recordings}

Stimuli were presented using Psychopy ver. 1.82.01 (Peirce, 2007) on a Windows desktop computer. Subjects were seated in a chair at a desk with their heads stabilized on a chin rest. Responses were recorded using force sensors (Phidgets, Inc., Calgary, Canada) attached to a computer keyboard. Force sensors were used in order to maximize temporal resolution of responses and were attached to the computer keyboard to allow familiar tactile feedback for the subjects and to facilitate the calculation of RT and error rate between blocks as immediate feedback for each subject. The stimulus monitor was positioned at eye level, $20 \mathrm{~cm}$ away, while the keyboard with attached force sensors was placed at the most comfortable position for each subject. Real- time PD was measured using an EyeLink 1000 system (SR Research, Ottawa, Canada), recording at $1000 \mathrm{~Hz}$, with pupil diameter measured in arbitrary units. All stimuli and responses were synchronized and stored using the ioHub Event Monitoring Framework within the PsychoPy package.

\subsection{Analysis}

\subsubsection{Behavioral analysis}

2.4.1.1. Reaction times. For immediate feedback following each block, $\mathrm{RT}$ was measured as the difference in time between stimulus onset and the first keyboard press within $1.5 \mathrm{~s}$ of each trial. For all further analyses, reaction time was defined as the difference in time between stimulus onset and $30 \mathrm{~g}$ of force preceding the first keyboard press within $1.5 \mathrm{~s}$. The value of $30 \mathrm{~g}$ was empirically determined to be the minimum amount of force required to engage a button press on that particular computer keyboard. This allowed for millisecond-precision timing of responses. Any trials that did not include a physical keyboard button press were not included in the analysis.

2.4.1.2. Response types. Response types were defined as follows, based on the unique combinations of stimuli and responses.

Main response types:

- Pattern Go (PGo): correct Go response (right index) to the Go stimulus (the digits 1,2 , or 4 ) matching the repeated pattern. I.e., occurring in the expected location within the sequence.

- Pattern Switch (PSwitch): correct switch response (left index) to the probable Switch stimulus (the digit 3) matching the repeated pattern.

- Deviant Go (DGo): correct go response (right index) to a Go stimulus (digit 1, 2, or 4) deviating from the repeated pattern, i.e., a Go stimulus where an expected " 3 " stimulus would have occurred requiring a Switch to a left index response. For example, for a subject responding to sequence A (3-1-4-3-2-4-1-2), a sample stream of stimuli with a Deviant Go trial (underlined) would be: 3-1-4-1-2-4-12. If the Pattern stimulus was expected, a change from a Switch to a Go response was required.

- Deviant Switch (DSwitch): correct switch response (left index) to improbable switch " 3 " stimulus, deviating from the repeated sequence, i.e., where the expected Pattern stimulus would have required a Go response. For example, for a subject responding to sequence A (3-1-4-3-2-4-1-2), a sample stream of stimuli with a Deviant Switch trial (underlined) would be: 3-1-4-3-2-3-1-2. For this response type, if the Pattern stimulus was expected, a change from a Go to a Switch response was required.

Additional response types:

- Explicit Go (ExGo): correct Go (right index) response to Pattern stimulus 1, 2, or 4 occurring within the given sequence, immediately following a " 3 " stimulus. This response type was analyzed separately from other Go responses, as subjects quickly learned that the Switch stimulus " 3 " never occurred twice in succession and therefore could explicitly predict that a Go trial would follow a Switch trial.

- Go Error (Err(Go)): failure to perform the Go response, defined as any Switch (left index) response to a Pattern or Deviant Go stimulus ("1", "2" or "4").

- Switch Error (Err(Sw)): failure to perform the Switch response, i.e., a Go (right index) response to a Pattern or Deviant Switch stimulus (“3”).

Any missed trials where subjects did not respond within $1.5 \mathrm{~s}$ of stimulus presentation were rare and not included in any analyses.

2.4.1.3. Efficiency. Efficiency of each subject's performance was determined by calculating the accuracy (percent correct) and dividing 


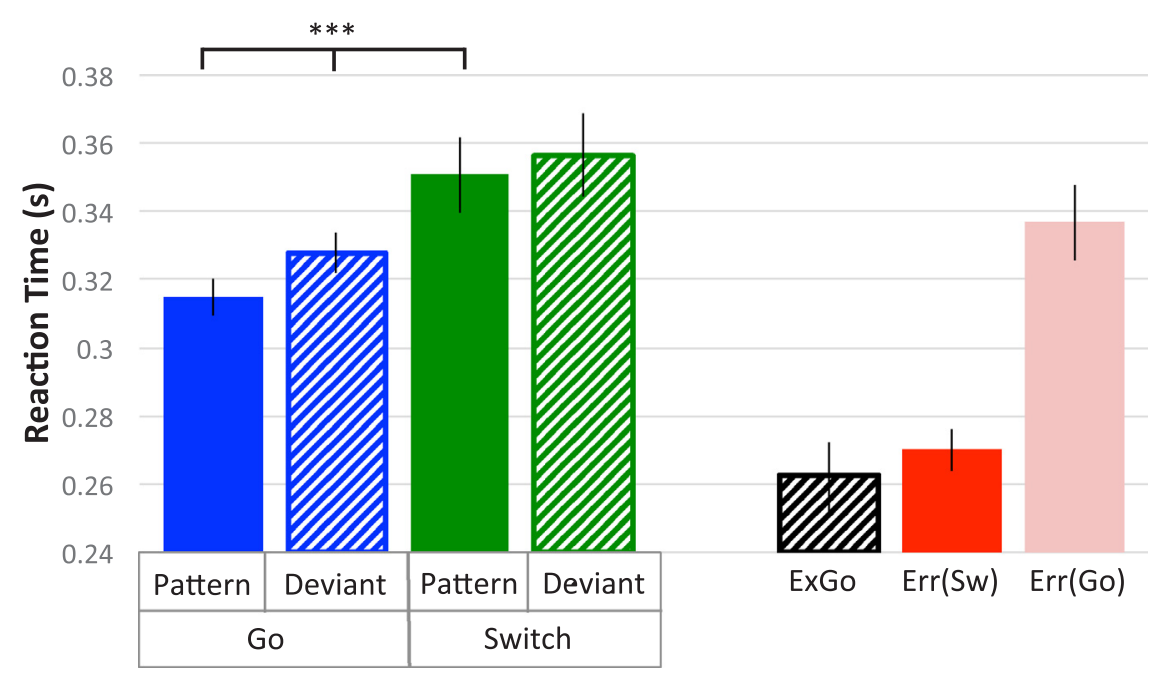

Fig. 2. Reaction time. Mean and standard errors for response types Pattern and Deviant Go, Pattern and Deviant Switch, Explicit Go, and Errors (Switch and Go, respectively). Pattern Go, Deviant Go and Pattern Switch response types were all significantly different from each other (all $p<0.001$ ), but not Pattern and Deviant Switch $(p=0.14)$. by the reaction time over the entire experiment for each of the 4 main trial types (PGo, PSwitch, DGo, DSwitch).

\subsubsection{Pupil diameter}

Continuously recorded PD data was segmented into epochs and time-locked to stimulus onset. Eye blinks were linearly interpolated using a custom Matlab script, bandpass filtered from 0.01 to $10 \mathrm{~Hz}$, and then z-transformed within participants to minimize inter-subject variability.

It was identified by Beatty and Lucero-Wagoner that there are three TEPRs that typically intensify as a function of cognitive load: mean pupil dilation, maximum pupil dilation, and latency to maximum (TMax) (Beatty and Lucero-Wagoner, 2000). In addition, we also measured here the pre-stimulus pupil diameter.

Pre-stimulus pupil diameter was measured as the mean z-scored pupil diameter for the $0.4 \mathrm{~s}$ preceding stimulus onset, which was then subtracted from the entire trial. TEPR was measured as the mean zscored pupil diameter for the $2.0 \mathrm{~s}$ following stimulus onset (until the subsequent pre-stimulus time period).

\subsubsection{Statistical analyses}

RT was log-transformed to normalize its distribution. To determine whether there was an effect of the probabilistic sequence pattern on either Go or Switch responses across test blocks on error rates, $\operatorname{logRT}$ and the various PD measures, repeated measures ANOVAs were conducted, and any post-hoc comparisons were conducted using $t$-tests with Bonferroni corrections. To determine effect sizes of statistically significant ANOVA results, the following formula was used (Lakens, 2013):

$\eta_{p}^{2}=\frac{F^{*} d f_{1}}{\left(F^{*} d f_{1}\right)}+d f_{2}$

where $\eta_{p}{ }^{2}$ is partial eta-squared, $F$ is the F-statistic, $d f_{1}$ is the effect degree of freedom, and $d f_{2}$ is the error degree of freedom. To determine effect sizes of statistically significant post-hoc paired $t$-test results, the following formula was used (Lakens, 2013):

$E S=\frac{m_{D I F F}}{S_{D I F F}}$

where

$S_{\text {DIFF }}=\sqrt{s_{1}^{2}+s_{2}^{2}-2 \times r \times s_{1} \times s_{2}}$

and $E S$ is the dimensionless Cohen's $d_{z}, m_{D I F F}$ is the mean of the difference between conditions, $S_{D I F F}$ is the standard deviation of the difference between conditions, $s_{1}$ and $s_{2}$ are the standard deviation of each condition, and $r$ is the correlation coefficient.

\section{Results}

\subsection{Behavioral results}

All subjects complied with task instructions, completed all 7 blocks and provided feedback on the task. All subjects earned the maximum $\$ 14$ performance reward. None of the subjects were able to replicate the complete stimulus sequence at the end of the experiment, and failed to provide any evidence of explicit knowledge of the stimulus sequences.

\subsubsection{Reaction times}

To determine the effects of the task parameters (Go/Switch and Pattern/Deviant) on response speed, we analyzed mean log-transformed reaction times. A 2-way ANOVA was conducted for PGo, DGo, PSwitch, DSwitch, revealing a significant main effect of response hand $\left(\mathrm{F}(1,11)=13.39, p=0.004\right.$, effect size $\left.\eta_{p}{ }^{2}=0.55\right)$ and of pattern ( $\mathrm{F}$ $(1,11)=15.59, p=0.002$, effect size $\left.\eta_{p}{ }^{2}=0.59\right)$ with no significant interaction $(\mathrm{F}(1,11)=2.32, p=0.16)$. Post-hoc comparisons revealed significant differences between PGo and PSwitch $(p<0.002$, effect size $\left.d_{z}=1.18\right)$, as well as PGo and DGo $\left(p<0.007\right.$, effect size $d_{z}=$ 1.43), but not PSwitch and DSwitch $(p=0.25)$. Mean RT ( \pm standard error) was greater for Switch responses over Go, and greater for Deviant over Pattern trials (PGo $=0.315 \pm 0.003 \mathrm{~s}$, PSwitch = $0.351 \pm 0.005 \mathrm{~s}, \quad$ DGo $=0.328 \pm 0.003 \mathrm{~s}$, DSwitch $=$ $0.356 \pm 0.006 \mathrm{~s}$ Fig. 2). Response speeding was observed for Switch Error trials $(\mathrm{Err}(\mathrm{Sw})=0.276 \pm 0.003 \mathrm{~s})$ and trials following the target "3" stimulus (ExGo $=0.262 \pm 0.005 \mathrm{~s}$ ). Response speeding was not observed for Go Error trials $(\operatorname{Err}(\mathrm{Go})=0.333 \pm 0.006 \mathrm{~s})$.

\subsubsection{Efficiency}

To determine whether the task parameters (Go/Switch and Pattern/ Deviant) had effects on overall performance, we analyzed mean efficiency rates (accuracy/reaction time) for each trial type. A 2-way ANOVA was conducted for PGo, DGo, PSwitch, DSwitch, revealing a significant main effect of response hand $(\mathrm{F}(1,11)=56.81, p<0.001$, effect size $\left.\eta_{p}{ }^{2}=0.84\right)$ and of pattern $(\mathrm{F}(1,11)=24.45, p<0.001$, effect size $\eta_{p}{ }^{2}=0.69$ ) with a significant interaction between the two ( $\mathrm{F}$ $(1,11)=5.20, p=0.04$, effect size $\left.\eta_{p}{ }^{2}=0.32\right)$. Post-hoc comparisons revealed significant differences between PGo and DGo $(p<0.001$, effect size $\left.d_{z}=1.40\right)$, as well as PSwitch and DSwitch $(p<0.002$, effect size $d_{z}=2.31$ ). The interaction reveals a larger effect of Deviant when paired with Switch as follows: mean Efficiency ( \pm standard error) was greater for Go responses over Switch, and greater for Pattern over Deviant trials (PGo $=3.12 \pm 0.05$ correct $/ \mathrm{s}$, PSwitch $=$ $2.29 \pm 0.12 \mathrm{correct} / \mathrm{s}, \mathrm{DGo}=3.03 \pm 0.06 \mathrm{correct} / \mathrm{s}$, DSwitch $=$ $2.04 \pm 0.14$ correct/s; Fig. 3). 


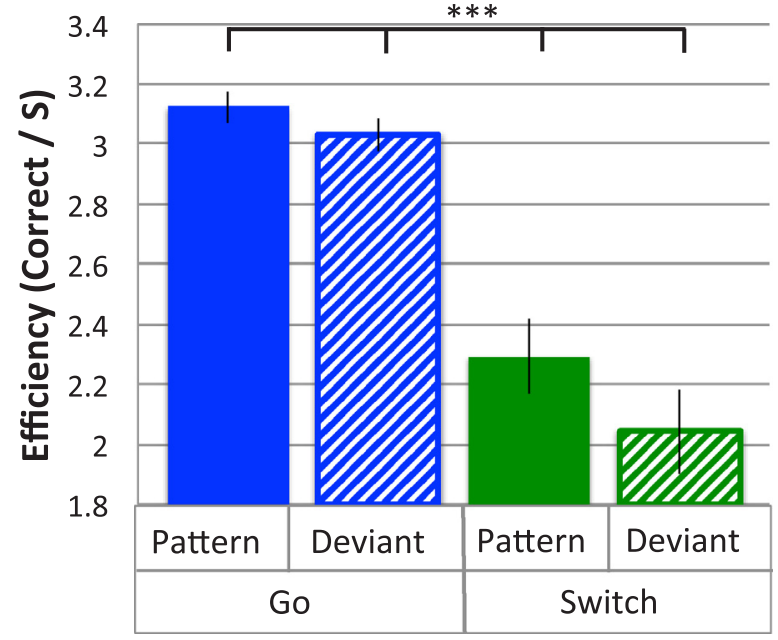

Fig. 3. Efficiency Rates across trial types (Correct/s). Efficiency is the accuracy of each response divided by its RT, or, speed of correct responding. Mean and standard errors for response types Pattern and Deviant Go, and Pattern and Deviant Switch. All 4 trial types were significantly different from each other $(p<0.002)$.

\subsection{Physiological measures}

\subsubsection{Pupil diameter}

Pupil diameter followed a typical time course, beginning at a minimum prior to stimulus onset, and dilating to a maximum diameter within $0.5-1.5 \mathrm{~s}$ (Fig. 4). Pupil diameters generally returned to approximately pre-stimulus levels ahead of the next trial at $2.4 \mathrm{~s}$.

\subsubsection{Task-evoked pupil responses}

Three types of task-evoked pupil responses (TEPR) are known to increase with cognitive load. The most commonly described TEPR is mean dilation (mTEPR). In order to determine the effects of the task parameters (Go/Switch and Pattern/Deviant) on this physiological response, TEPR was calculated as the mean baselined z-scored pupil diameter for $2 \mathrm{~s}$ following stimulus onset (time 0-2 s; Fig. 4). A 2-way ANOVA was conducted on mean TEPR for PGo (mean $=0.64 \pm 0.053$ $\mathrm{z})$, PSwitch (mean $=0.96 \pm 0.051 \mathrm{z})$, DGo $($ mean $=0.79 \pm 0.052 \mathrm{z}$ ), and DSwitch (mean $=0.92 \pm 0.079 \mathrm{z}$ ). The ANOVA revealed a statistical main effect of response hand $(\mathrm{F}(1,11)=46.61, p<0.001$, effect size $\left.\eta_{p}{ }^{2}=0.81\right)$ but not of pattern $(\mathrm{F}(1,11)=3.56, p=0.086$, effect size $\left.\eta_{p}{ }^{2}=0.24\right)$ with a significant interaction between the two ( $F$ $(1,11)=6.21, p=0.03$; Fig. 5). Post-hoc comparisons revealed

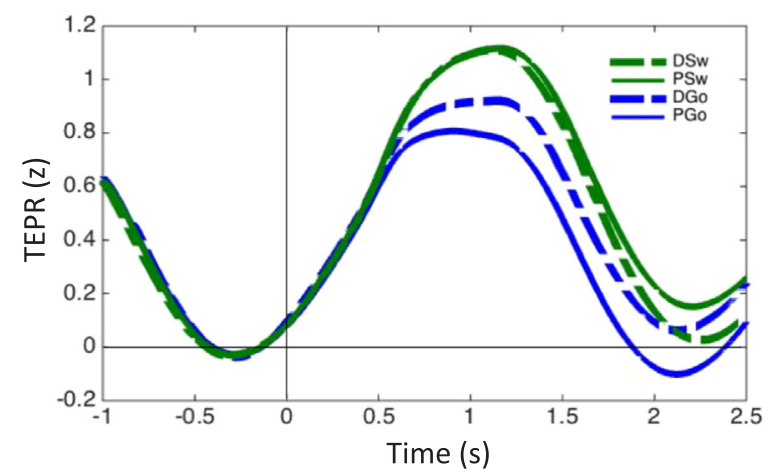

Fig. 4. Pupil Diameter Time Course. Pupil diameter is within-subject z-scored, and time-locked to cue onset. Pre-Stimulus PD for each trial was calculated as the average z-score value within the $0.4 \mathrm{~s}$ preceding stimulus onset, and was subtracted from the entire trial. Task-evoked pupil response for each trial was calculated as the average $\mathrm{z}$-score value over $2.0 \mathrm{~s}$ following stimulus presentation. significant differences between Pattern and Deviant Go $(p=0.005$, effect size $\left.d_{z}=0.79\right)$, and Pattern Go and Switch $(p<0.001$, effect size $\left.d_{z}=1.71\right)$ but not Pattern and Deviant Switch $(p=0.24)$. Reduced TEPR was observed for ExGo (mean $=0.46 \pm 0.07 \mathrm{z}$ ), whereas increased TEPR followed Go and Switch Errors (mean Err(Sw) $=1.11 \pm 0.10 \mathrm{z}$, mean $\operatorname{Err}(\mathrm{Go})=1.18 \pm 0.13 \mathrm{z}$ ). The results of the second TEPR, maximum dilation, were in no measurable way different from mean dilation, and therefore not included in any further analysis.

The third TEPR, latency to maximum dilation (T-Max), was of additional interest given the known relationship between TEPR and brain rhythms. T-Max was calculated as the mean latency of maximum pupil dilation following stimulus onset (Fig. 6). A 2-way ANOVA was conducted on mean pupil T-Max for PGo (mean $=1.00 \pm 0.08 \mathrm{~s}$ ), PSwitch $($ mean $=1.10 \pm 0.08 \mathrm{~s})$, DGo (mean $=1.00 \pm 0.08 \mathrm{~s})$, and DSwitch (mean $=1.05 \pm 0.048 \mathrm{~s}$ ). The ANOVA revealed a statistical main effect of response hand only $\left(\mathrm{F}(1,11)=9.21, p=0.01\right.$, effect size $\eta_{p}{ }^{2}$ $=0.46$; Fig. 6 ). Delayed pupil T-Max was observed for $\operatorname{Err}(\mathrm{Sw})(\mathrm{mean}=$ $1.19 \pm 0.05 \mathrm{~s})$ and $\operatorname{Err}(\mathrm{Go})($ mean $=1.26 \pm 0.07 \mathrm{~s})$.

\subsubsection{Comparing reaction time and mean task-evoked pupil response}

In order to determine whether the outcome measures were measuring different underlying processes, a 3-way ANOVA was conducted on two outcome measures (logRT and mTEPR) as well as the task parameters (Go/Switch and Pattern/Deviant). Contrary to results above, all main effects were statistically significant (measure, response hand and pattern, all $\mathrm{F}(1,11)>12.3$, all $p<0.005$, all effect size $\eta_{p}{ }^{2}$ $\geq 0.53$ ). Furthermore the following interactions were significant: hand $\mathrm{x}$ pattern $\left(\mathrm{F}(1,11)=8.89, p=0.013\right.$, effect size $\eta_{p}{ }^{2}=0.45$; Fig. 7 ).

\subsubsection{Pre-stimulus pupil diameter}

The pupil diameter during the pre-stimulus period was measured as the average z-score during the $0.4 \mathrm{~s}$ prior to stimulus onset. To determine whether the stimulus pattern had any effect on pre-stimulus minimum pupil diameter, a student's $t$-test was conducted on averaged PD during the $0.4 \mathrm{~s}$ prior to stimulus onset comparing PGo and PSwitch trials. There was no statistical difference between Pattern trial types $(p=0.18$; data not shown $)$.

\section{Discussion}

In the current study we compared how stimulus probability effects cognitive load and behavioral responses by combining a Go/Switch task with incidental pattern learning of fixed sequences in stimulus presentation, where mTEPR was used as an indicator of cognitive processing.

\subsection{Pattern learning facilitates performance}

Differences between Pattern and Deviant trials in terms of RT, Efficiency and mTEPR demonstrated clear evidence of subjects learning the stimulus pattern. The existence of the Pattern facilitated performance in terms of decreased RT in Pattern over Deviant trials (Fig. 3), as well as increased the efficiency of Go and Switch responses (Fig. 2). In order to exclude any effects prior to pattern learning, all analysis was repeated for blocks 2-7 only and excluding block 1, with no significant differences in the results. In addition, at the end of the experiment, no subject reported any subjective differences across blocks, and none were able to report the stimulus sequence. A surprising result was that a change in sequences in Block 6 did not appear to affect task performance. This suggests that what was learned was not the specific order of stimuli, which varied across the two sequences, but rather motor learning of the numbers of Go trials between Switch trials (2 or 4), which were the same for both sequences used. This is consistent with the finding that pattern sequences predominantly involve motor learning of required responses over perceptual learning of stimuli (Deroost and Soetens, 2006). In the current study, what subjects likely 


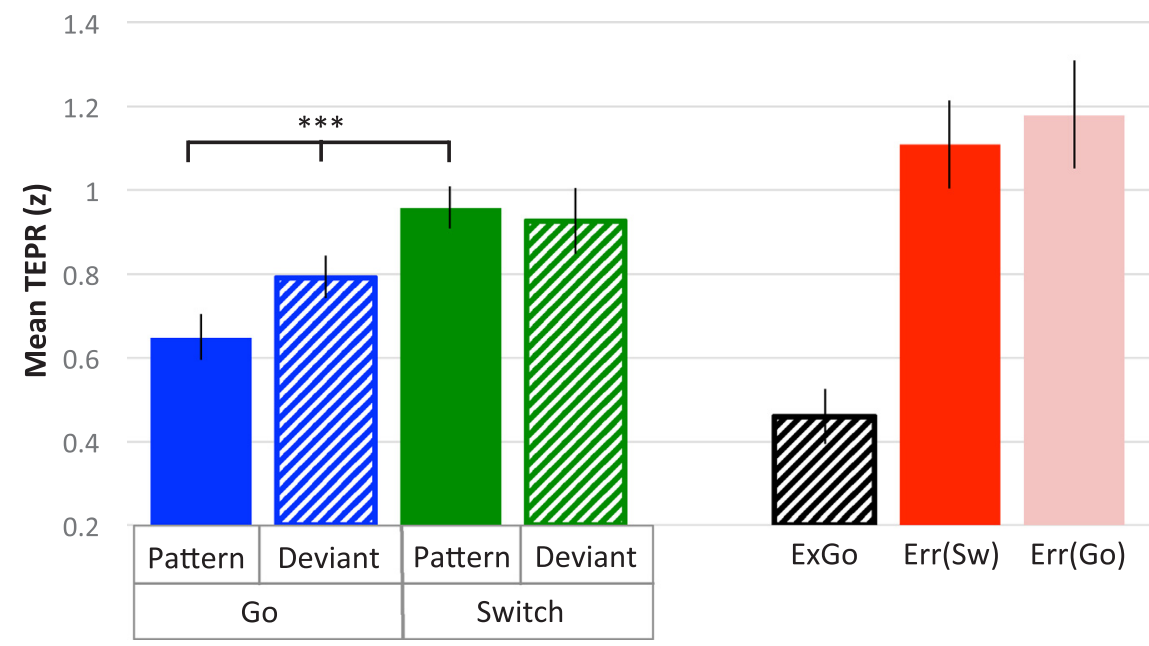

Fig. 5. Task-Evoked Pupil Response. Mean TEPR (in z-scores) and standard errors for response types Pattern Go, Pattern Switch, Deviant Go, Deviant Switch, Explicit Go, and Errors (Switch and Go, respectively). All response types were significantly different (all $p<0.001$ ) except for PSwitch and DSwitch $(p=0.36$ ).

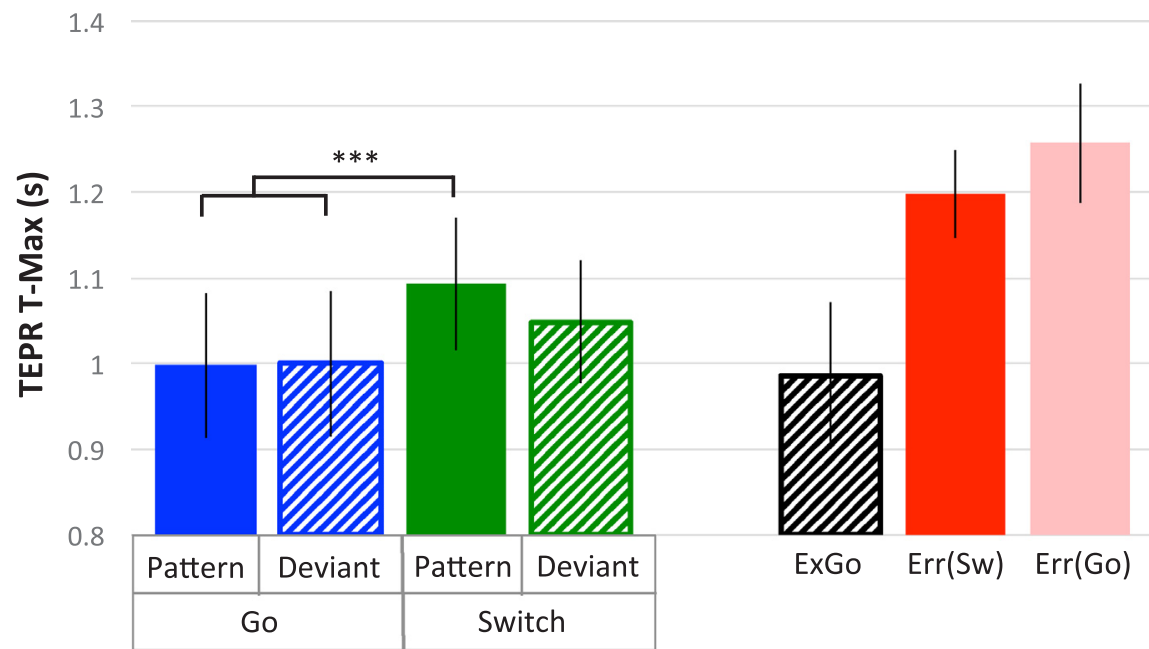

Fig. 6. Time to Peak Task-Evoked Pupil Response. Time (in seconds) and standard errors for response types Pattern Go, Pattern Switch, Deviant Go, Deviant Switch, Explicit Go, and Errors (Switch and Go, respectively). Go and PSwitch responses were significantly different $(p<0.001)$ but not Pattern and Deviant $(p>0.18)$.

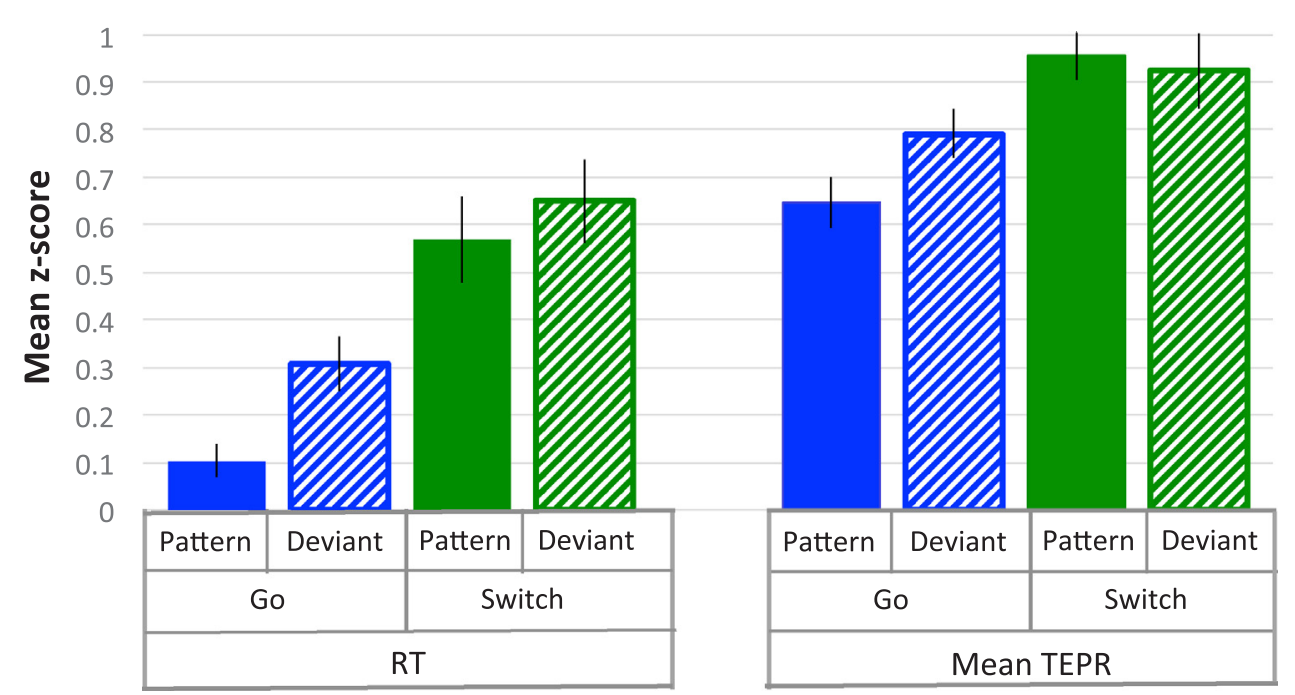

Fig. 7. RT and TEPR. Mean RT and TEPR with standard errors for response types Pattern Go, Pattern Switch, Deviant Go, Deviant Switch. All data is within-subject z-scored. All main effects were statistically significant (Measure (RT-TEPR), Hand (Go-Switch) and Pattern, all $\mathrm{F}(1,11)>12.3$, all $p<0.005)$. There were significant interactions between Hand and Pattern, $(\mathrm{F}(1,11)=8.89, p=0.013)$. 
learned was the degree to which they should prepare a Go or a Switch response ahead of the upcoming trial based on the length of intervals between Switch trials. These results are congruent with those of (Deroost et al., 2012) when response inhibition was combined with incidental sequence learning to manipulate cognitive control in a Stroop task. Unbeknownst to the subjects, the order of stimuli followed a specific grammar, which led to facilitation of task performance, but no reduction in response conflict. It is likely that although unconscious awareness of an upcoming incongruent trial did not reduce the colorword conflict associated with the Stroop effect, the learned stimulus order did reduce RT and likely facilitated response preparation in both the Stroop and the current SART tasks.

\subsection{T-Max reveals latency of response selection}

Differences in T-Max were limited to Go and PSwitch trials, which are a prepotent and relatively rare response, respectively. T-Max was not, however, sensitive to the stimulus pattern. A previous study shows that the latency of pupil dilation reveals the timing of button response selection (Einhauser et al., 2010). Applying this interpretation of T-Max to the present results suggest that the time to select a button response was contingent upon the overall probability of Go (75\%) and Switch (25\%) response requirements, and not contingent upon conditional probability defined by the pattern $(90 \%)$. In contrast, considering that mTEPR may reflect processing load, a difference in mTEPR for PGo and DGo responses without a similar difference in T-Max suggests that there could have been a difference in processing load in the absence of a difference in time to select a response. Furthermore, T-Max was not sensitive to the pattern whereas RT was, suggesting a distinction between response selection and response execution. This finding has important implications for the neural control of movement, and warrants replication and further study.

\subsection{RT and mTEPR: indexes of processing latency and load?}

We found significant main effects of response hand (Go/Switch) across $\operatorname{logRT}$ and mTEPR and stimulus pattern (Pattern/Deviant) for $\operatorname{logRT}$ only. Upon further inspection, although there is an effect of stimulus pattern on mTEPR for DGo (and not DSw), there is a much larger effect of response hand on mTEPR (effect size $\eta_{p}{ }^{2}=0.77$ ) than on RT (effect size $\eta_{p}{ }^{2}=0.55$ ). Further to the difference in effects of Go/Switch on RT and MTEPR, differences between these two measures have been observed previously (Richer et al., 1983; Molen et al., 1989; Hyönä et al., 1995), and it was asserted that they might index different cognitive processes, in that RT may index processing latency whereas mTEPR may index processing load (Richer and Beatty, 1987).

Deviant and Switch trials had increased mTEPR and increased RT over PGo trials, but the relative amounts were not equal in terms of percent changes. Deviant Go stimuli led to a $18 \%$ increase in mTEPR (mean $=0.78 \mathrm{z}$ ) over PGo $(0.64 \mathrm{z})$, whereas Switch stimuli led to a $36 \%$ increase in mTEPR $(1.00 \mathrm{z})$. In contrast, effects of Deviant and Switch on RT was much smaller and more similar: Deviant Go stimuli were associated with a $4 \%$ increase in RT (mean $=0.33 \mathrm{~s}$ ) over PGo ( $0.31 \mathrm{~s})$, whereas Switch stimuli led to an $11 \%$ increase in RT $(0.35 \mathrm{~s})$. Based on these differences, mTEPR was more sensitive to the Go/Switch parameter than RT. Responding to a Switch (either P or D) stimulus is associated with much greater mTEPR than responding to a Deviant Go stimulus. Congruent with research on mTEPR, we interpret this finding as increased cognitive load requirements between Go and Switch responses. In contrast, Deviant Go stimuli caused increased RT, and Switch stimuli caused an additional increase in RT, approximately doubly sized. Contrary to previous theory (Richer and Beatty, 1987), we do not interpret RT as having indexed processing latency, but rather as response execution. As was discussed above, we consider that latency to process the stimuli and select a response was indexed by T-Max. We suggest these theories should be confirmed with functional brain imaging data.

Increased TEPR has been found to occur following surprise signals (Preuschoff et al., 2011). We interpret Deviant Go responses in light of being 'surprised', in the sense that the stimulus was not what subjects expected from the pattern. However, percent increase in mTEPR over PGo trials was 2-times larger for Switch responses (36\%) than for Deviant Go responses (15\%), even though Pattern Switch responses should have been expected. We interpret this result in terms of response preparation: subjects did not prepare Switch responses to the same degree as they prepared Go responses, despite pattern learning. This result is in line with previous work on the SART and response switching tasks, demonstrating that Go responses quickly become "prepotent" and automatic (Cheyne et al., 2012). Furthermore, relatively larger effect sizes of Switch responses on mTEPR over RT suggests that execution of the less prepotent Switch response requires greater load to execute, without a corresponding increase in RT. An increase in load without an increase in RT may be cause by increased cognitive effort by the participants, where effort is a measure of load over time.

\subsection{Efficiency}

Performance efficiency, defined as accuracy / reaction time, is the only measure to distinguish pattern and deviant Switches. We suggest this could be a reflection of speed-accuracy strategy, in that subjects maintained speed of responding to Switch stimuli, at a cost of accuracy during deviant trials. Performance efficiency takes into account differences in speed-accuracy strategy and integrates both parameters into a single value. We speculate that this measure is likely closest to a behavioral measure of cognitive effort in this study.

\section{5. mTEPR relates to neural activity}

That greater processing load is required for Switch responses over Go responses is supported by results from previous studies indicating increased frontal theta (4-8 Hz) power for Switch over Go trials, which is thought to reflect top-down inhibitory processing (Cheyne et al., 2012; Isabella et al., 2015). Why the pattern would facilitate greater efficiency but not faster RT or greater mTEPR for Switch trials, may arise because subjects were attempting to achieve rapid responses rather than avoiding an error on DSw trials. It is possible that it was easier for participants to respond more quickly to the pattern during Go trials without a significant decrease in accuracy, but it was more difficult to increase speed without sacrificing accuracy for Switch trials.

Results from the additional response types were consistent with this notion of distinguishing mTEPR and RT, based on their differential sensitivity to cognitive load. For example, relative to other trial types, Err(Sw) responses showed a fast RT (Fig. 3) and large mTEPR (Fig. 5), indicating large processing load. It is known from previous work that $\operatorname{Err}(\mathrm{Sw})$ responses tend to be executed with very short latencies and are accompanied by large increases in brain activity, including frontal theta oscillations, likely related to cognitive processing following the response (Cheyne et al., 2012; Isabella et al., 2015). In this study, large mTEPR following an error response is likely also related to cognitive processing. In contrast, it was clear from the current results that Err(Go) was a very different type of error, in that although RT was moderately slow compared with $\operatorname{Err}(\mathrm{Sw}), \operatorname{Err}(\mathrm{Go})$ was a rather fast Switch response compared with the other (Pattern and Deviant) Switches. Errors occurred with a relatively fast RT compared to correct responses of the same type. Furthermore, mTEPR was relatively large for Err(Sw) over correct Switches, indicating increased processing load. Lastly, ExGo shows consistently fast RT, even when compared with PGo, and also small mTEPR. Given that ExGo trials were explicitly learned, we interpret small TEPR as reflecting minimal processing requirements, leading to fast RTs and efficient responding. This is in direct contrast with the rest of the patterned stimuli (i.e., PGo and PSwitch).

The foregoing interpretations of the present data are supported by 
several studies that show mTEPR is closely linked with functional brain measures, and to a greater extent than RT. MTEPR has been linked to cortical surface negativity during response preparation (Jennings et al., 1998), and concurrent EEG measures have provided evidence that mTEPR can be a good predictor of changes in brain measures related to cognitive load and task difficulty, including alpha and frontal theta (Scharinger et al., 2015a, 2015b). This is further highlighted in a study reporting a linear relationship between pre-stimulus alpha power and mTEPR, but not RT (Hong et al., 2014). This suggests that Pattern and Deviant Go and Switch responses may produce differences not only in mTEPR, but also functional brain measures associated with processing load.

In addition to frontal activity, pupil diameter is tightly linked with activity in the locus coeruleus (LC) (Joshi et al., 2016) and is associated with activation of noradrenergic pathways in patients (Jepma et al., 2011), while in rodents it is associated with noradrenergic and cholinergic pathways (Reimer et al., 2016). Furthermore, baseline pupil size and pupil responses during cognitive processing have been shown to be related to the BOLD signal and to LC activity (Murphy et al., 2014; de Gee et al., 2017). In addition, Modafinil administration, which increases synaptic noradrenaline and dopamine, led to decreased taskindependent LC activity, increased task-related LC and prefrontal cortex activity, and enhanced LC-prefrontal cortex functional connectivity (Minzenberg et al., 2008). Subjects demonstrated increased cognitive performance and increased LC-mediated pupillary dilation. Furthermore, LC pathology is implicated in age-related cognitive decline (Mather et al., 2017). The current results suggest that mTEPR may reflect quantity, and T-Max may reflect latency of LC-mediated cognitive activity in the prefrontal cortex, albeit on a much longer time scale.

\section{Conclusions}

The methods used in this study are specifically related to differences in motor response preparation prior to the stimulus cue, which we directly relate to varying stimulus probabilities based on learning the stimulus sequences. We interpret the current findings as follows: RT is a measure of response execution, and MTEPR indexes cognitive load, and we conclude that subjects quickly learned the intervals between Switch trials imbedded in the stimulus pattern, leading to decreases in cognitive load required and latency response execution for PGo trials. In addition, if T-Max indexes response selection, and Efficiency indexes cognitive effort, then we conclude that although the stimulus pattern has no effect on response selection, both deviant and Switch trials have a significant effect on cognitive effort.

Cognitive load can be manipulated and measured in a variety of ways. Examples of measures of cognitive load include heart rate variability, task-evoked brain activity, eye activity (mTEPR or blink rate), or combinations of performance-based measures including RT, accuracy, and error rates (Paas et al., 2003). As noted there are limitations to performance-based measures of load, since mental effort can be increased in order to maintain consistent performance. By combining measures of cognitive load and performance-based measures in this study, we have been able to estimate the relative amounts of cognitive load and effort required for each trial type. Furthermore, the current task is simple and applicable to a range of populations. This study also highlights the advantages of using TEPRs as measures of cognitive load and latency, which can be different from typically used measures such as RT.

The data presented in this study show that modulating cognitive processing using pattern learning associated with differences in stimulus probabilities can impact RT, response efficiency, mTEPR, and TMax, all of which appear to index different cognitive processes underlying responding to a stimulus cue.

\section{Acknowledgements}

This work was supported by a Natural Sciences and Engineering Research Council of Canada Discovery Grant (\#184018-09).

\section{References}

Beatty, J., Lucero-Wagoner, B., 2000. The pupillary system. Handbook of Psychophysiology 2. pp. 142-162.

Cheyne, D.O., Ferrari, P., Cheyne, J.A., 2012. Intended actions and unexpected outcomes: automatic and controlled processing in a rapid motor task. Front. Hum. Neurosci. 6, 237.

Cheyne, J.A., Carriere, J.S., Smilek, D., 2009. Absent minds and absent agents: attentionlapse induced alienation of agency. Conscious Cogn. 18 (2), 481-493.

de Gee, J.W., Colizoli, O., Kloosterman, N.A., Knapen, T., Nieuwenhuis, S., Donner, T.H., 2017. Dynamic modulation of decision biases by brainstem arousal systems. Elife 6, e23232.

Deroost, N., Soetens, E., 2006. Perceptual or motor learning in SRT tasks with complex sequence structures. Psychol. Res. 70 (2), 88-102.

Deroost, N., Vandenbossche, J., Zeischka, P., Coomans, D., Soetens, E., 2012. Cognitive control: a role for implicit learning? J. Exp. Psychol.: Learn. Mem. Cognit. 38 (5), 1243-1258.

Einhauser, W., Koch, C., Carter, O.L., 2010. Pupil dilation betrays the timing of decisions. Front. Hum. Neurosci. 4, 18.

Gabriel, A., Maillart, C., Guillaume, M., Stefaniak, N., Meulemans, T., 2011. Exploration of serial structure procedural learning in children with language impairment. J. Int. Neuropsychol. Soc. 17 (2), 336-343.

Gehring, W.J., Gratton, G., Coles, M.G.H., Donchin, E., 1992. Probability effects on stimulus evaluation and response processes. J. Exp. Psychol. Hum. Percept. Perform. 18 (1), 198-216.

Hick, W.E., 1952. On the rate of gain of information. Q. J. Exp. Psychol. 4 (1), 11-26.

Hong, L., Walz, J.M., Sajda, P., 2014. Your eyes give you away: prestimulus changes in pupil diameter correlate with poststimulus task-related EEG dynamics. PLoS One 9 (3), e91321.

Hyönä, J., Tommola, J., Alaja, A.-M., 1995. Pupil dilation as a measure of processing load in simultaneous interpretation and other language tasks. Q. J. Exp. Psychol. Sect. A 48 (3), 598-612.

Isabella, S., Ferrari, P., Jobst, C., Cheyne, J.A., Cheyne, D., 2015. Complementary roles of cortical oscillations in automatic and controlled processing during rapid serial tasks. Neuroimage 118, 268-281.

Jacoby, L.L., Witherspoon, D., 1982. Remembering without awareness. Can. J. Psychol./ Rev. Can. Psychol. 36 (2), 300.

Jennings, J.R., van der Molen, M.W., Steinhauer, S.R., 1998. Preparing the heart, eye, and brain: foreperiod length effects in a nonaging paradigm. Psychophysiology 35 (1) 90-98.

Jepma, M., Deinum, J., Asplund, C.L., Rombouts, S.A., Tamsma, J.T., Tjeerdema, N., Spape, M.M., Garland, E.M., Robertson, D., Lenders, J.W., Nieuwenhuis, S., 2011. Neurocognitive function in dopamine-beta-hydroxylase deficiency. Neuropsychopharmacology 36 (8), 1608-1619.

Joshi, S., Li, Y., Kalwani, R.M., Gold, J.I., 2016. Relationships between pupil diameter and neuronal activity in the locus coeruleus, colliculi, and cingulate cortex. Neuron 89 (1), 221-234.

Kahneman, D., Beatty, J., 1966. Pupil diameter and load on memory. Science 154 (3756), 1583-1585.

Lakens, D., 2013. Calculating and reporting effect sizes to facilitate cumulative science: a practical primer for $t$-tests and ANOVAs. Front. Psychol. 4, 863.

Mather, M., Joo Yoo, H., Clewett, D.V., Lee, T.H., Greening, S.G., Ponzio, A., Min, J., Thayer, J.F., 2017. Higher locus coeruleus MRI contrast is associated with lower parasympathetic influence over heart rate variability. Neuroimage 150, 329-335.

Minzenberg, M.J., Watrous, A.J., Yoon, J.H., Ursu, S., Carter, C.S., 2008. Modafinil shifts human locus coeruleus to low-tonic, high-phasic activity during functional MRI Science 322 (5908), 1700-1702.

Molen, M.W., Boomsma, D.I., Jennings, J.R., Nieuwboer, R.T., 1989. Does the heart know what the eye sees? A cardiac/pupillometric analysis of motor preparation and response execution. Psychophysiology 26 (1), 70-80.

Murphy, P.R., O'Connell, R.G., O'Sullivan, M., Robertson, I.H., Balsters, J.H., 2014. Pupil diameter covaries with BOLD activity in human locus coeruleus. Hum. Brain Mapp. 35 (8), 4140-4154.

Nissen, M.J., Bullemer, P., 1987. Attentional requirements of learning: evidence from performance measures. Cogn. Psychol. 19 (1), 1-32.

Paas, F., Tuovinen, J.E., Tabbers, H., Van Gerven, P.W., 2003. Cognitive load measurement as a means to advance cognitive load theory. Educ. Psychol. 38 (1), 63-71.

Peirce, J.W., 2007. PsychoPy-psychophysics software in python. J. Neurosci. Methods $162(1-2), 8-13$.

Preuschoff, K., Hart, B.M. t., Einhauser, W., 2011. Pupil dilation signals surprise: evidence for noradrenaline's role in decision making. Front. Neurosci. 5, 115.

Reimer, J., McGinley, M.J., Liu, Y., Rodenkirch, C., Wang, Q., McCormick, D.A., Tolias, A.S., 2016. Pupil fluctuations track rapid changes in adrenergic and cholinergic activity in cortex. Nat. Commun. 7, 13289

Richer, F., Beatty, J., 1987. Contrasting effects of response uncertainty on the task-evoked pupillary response and reaction time. Psychophysiology 24 (3), 258-262.

Richer, F., Silverman, C., Beatty, J., 1983. Response selection and initiation in speeded reactions: a pupillometric analysis. J. Exp. Psychol.: Human. Percept. Perform. 9 (3), 360-370. 
Robertson, I.H., Manly, T., Andrade, J., Baddeley, B.T., Yiend, J., 1997. Oops!': performance correlates of everyday attentional failures in traumatic brain injured and normal subjects. Neuropsychologia. 35 (6), 747-758.

Scharinger, C., Kammerer, Y., Gerjets, P., 2015a. Pupil dilation and EEG alpha frequency band power reveal load on executive functions for link-selection processes during text reading. PLoS One 10 (6), e0130608.

Scharinger, C., Soutschek, A., Schubert, T., Gerjets, P., 2015b. When flanker meets the nback: what EEG and pupil dilation data reveal about the interplay between the two central-executive working memory functions inhibition and updating. Psychophysiology 52 (10), 1293-1304.
Seli, P., 2016. The attention-lapse and motor decoupling accounts of SART performance are not mutually exclusive. Conscious Cognit. 41, 189-198.

Simpson, H.M., Paivio, A., 1966. Changes in pupil size during an imagery task without motor response involvement. Psychon. Sci. 5 (10), 405-406.

Smallwood, J., Brown, K.S., Tipper, C., Giesbrecht, B., Franklin, M.S., Mrazek, M.D.,

Carlson, J.M., Schooler, J.W., 2011. Pupillometric evidence for the decoupling of attention from perceptual input during offline thought. PLoS One 6 (3), e18298.

Squires, N.K., Squires, K.C., Hillyard, S.A., 1975. Two varieties of long-latency positive waves evoked by unpredictable auditory stimuli in man. Electroencephalogr. Clin. Neurophysiol. 38 (4), 387-401. 